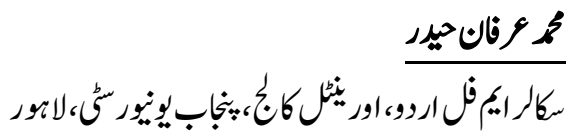

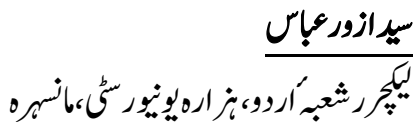

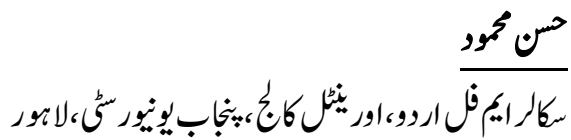

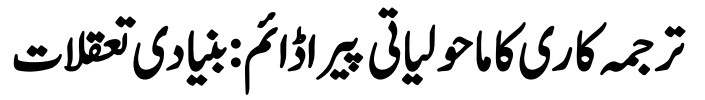

Muhammad Irfan Haider

Mphil Urdu Scholar 'Orientall College, Punjab University Lahore.

Syed Azwar Abbas

Lecturer of Urdu, Department of Urdu, Hazara University Mansehra.

Hassan Mehmood

Mphil Urdu Scholar, Orientall College, Punjab University Lahore.

\title{
Ecological Paradigm of Translation: Basic Concepts
}

Ecocriticism is an emerging discourse in literary studies. Ecocriticism, the study of human-nature relations in literature, film and other cultural expressions, has rapidly become established in the field of literary theory. Eco translatology is nature centered translation study and self-contained translation theory system, under the guidance of eco-holism. The recent cultural term in translation studies introduced a marked shift from strictly linguistic to cultural approches to translation. Eco translatology answers the fundamental questions in translation studies: what is translation? Who translates? How to translate and why to translate? In this article will be engaged all these prior mentioned questions and find their answers in the vision of eco criticism.

Keywords: Ecocriticism, Ecology, climate change, culture, Translation. 


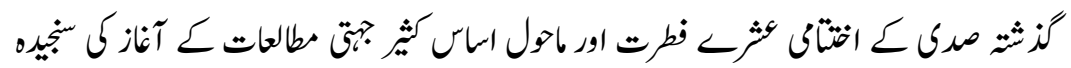

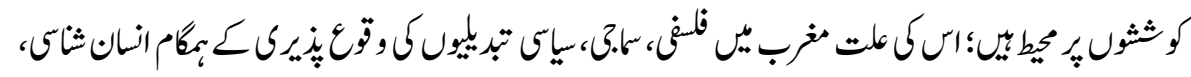

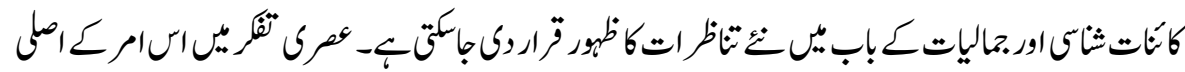

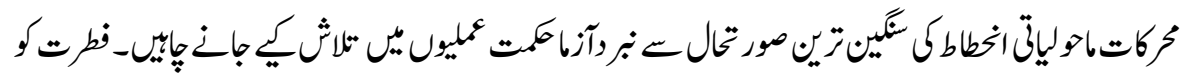

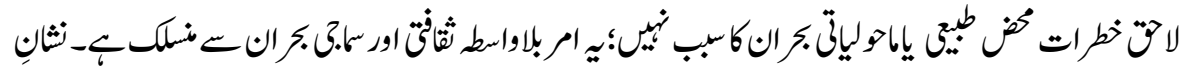

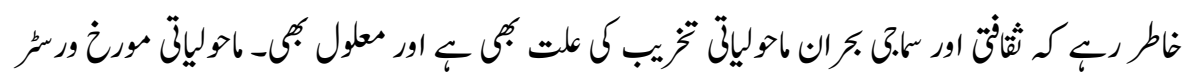
(Worster) (Climate Change)

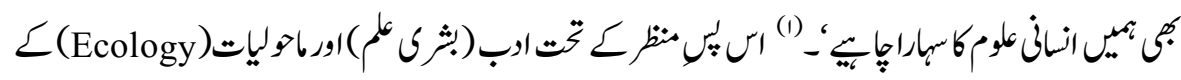

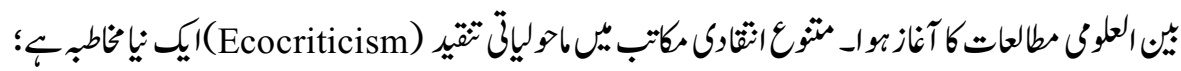

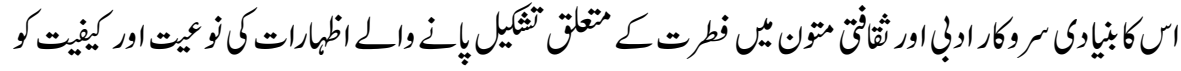

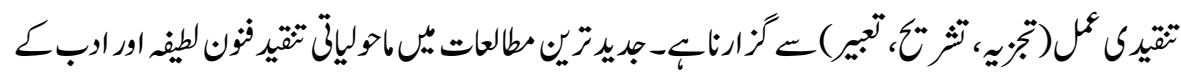

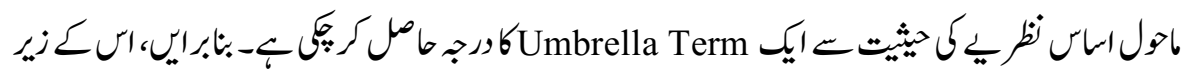

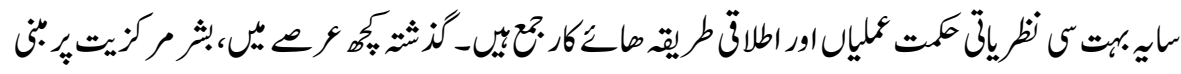

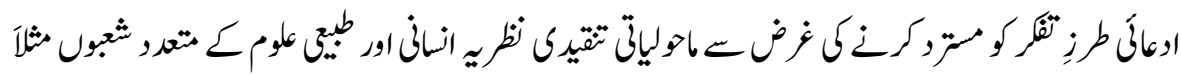

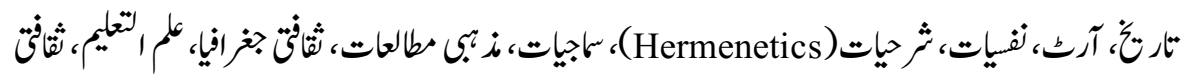

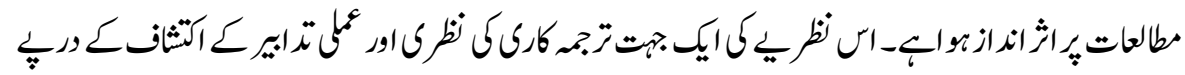

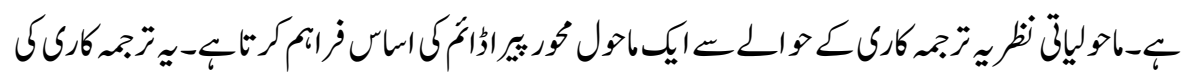

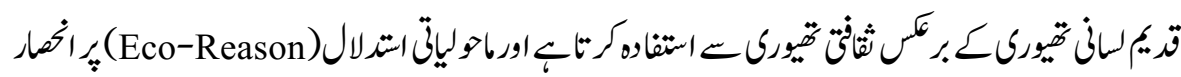

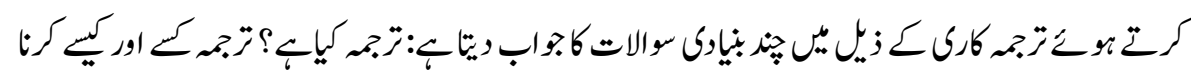

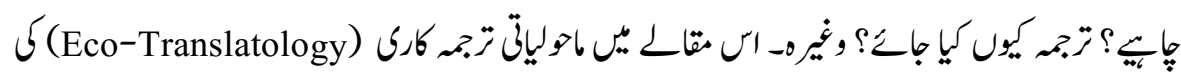

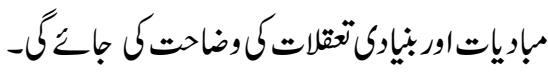




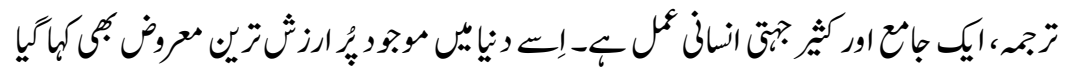

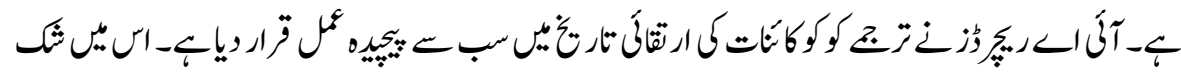

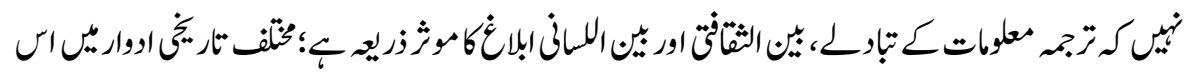

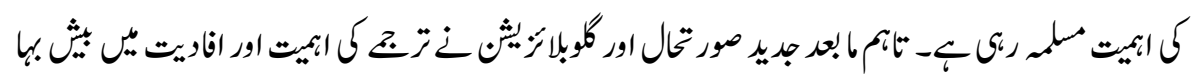

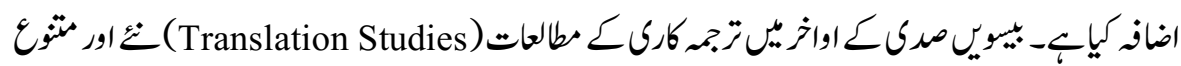

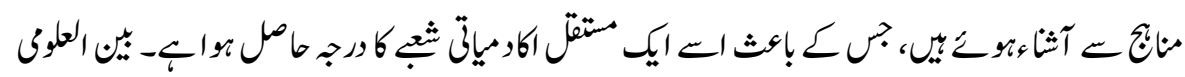
(Interdisciplinary)

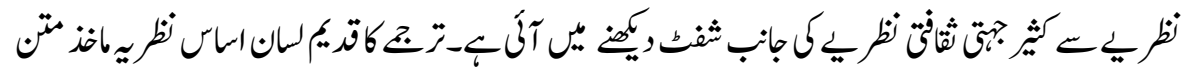
كوبن متثن (TT)

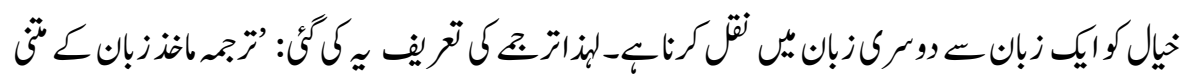

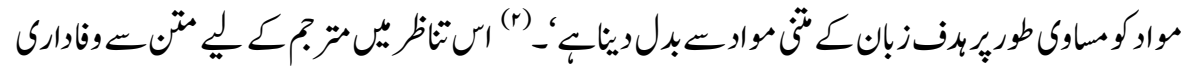

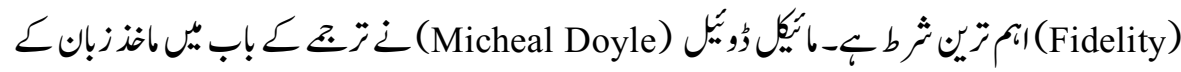

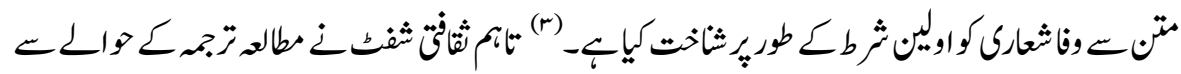

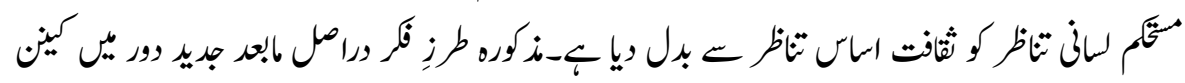
(Canon) اورثقانت) Cن (Translation, History and Culture

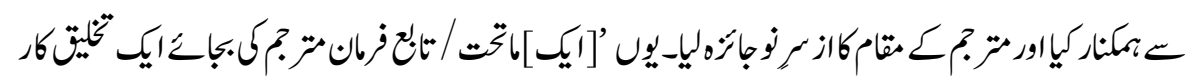

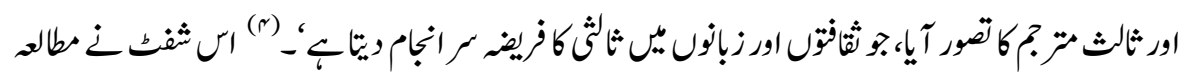

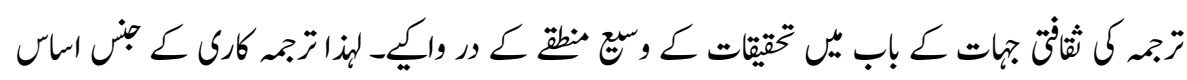
(Gender Studies)

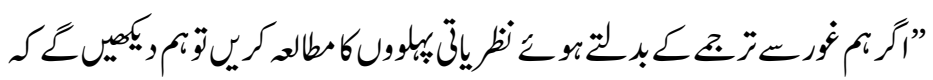

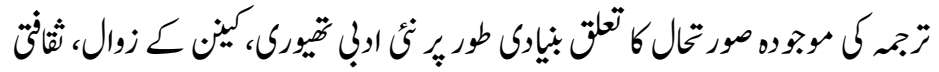




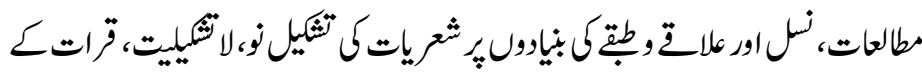

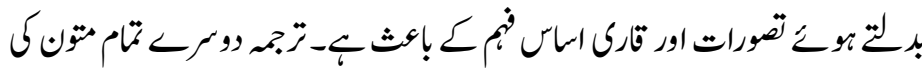

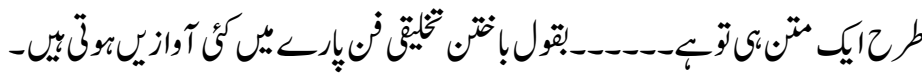

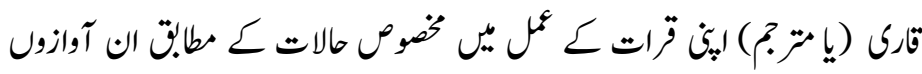

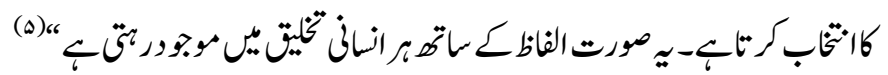

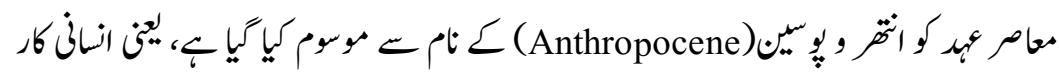

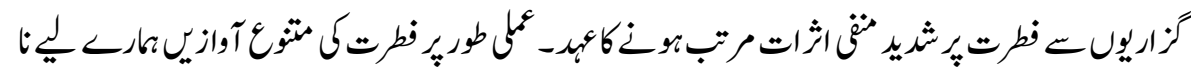

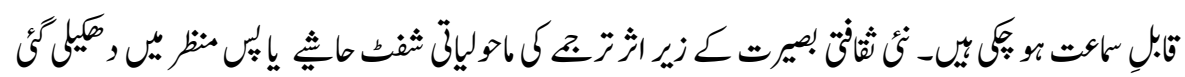

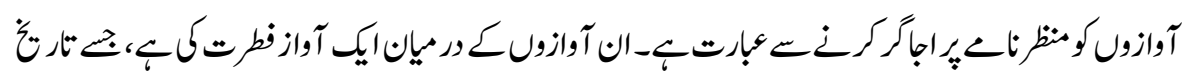

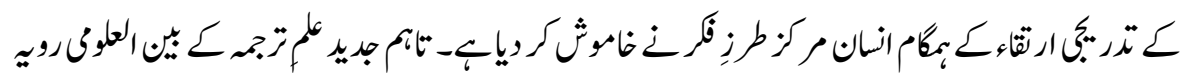

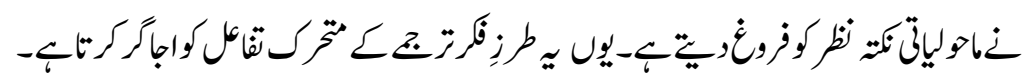

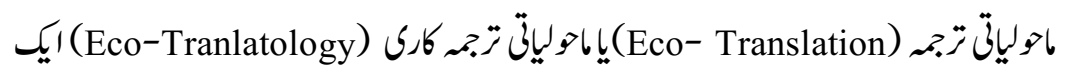

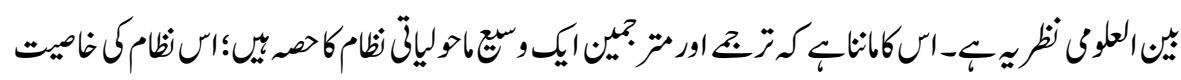

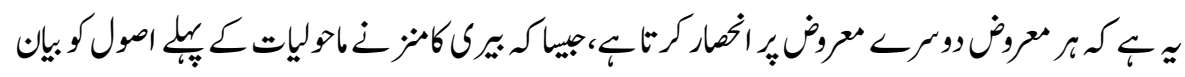

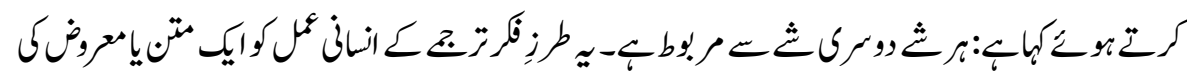

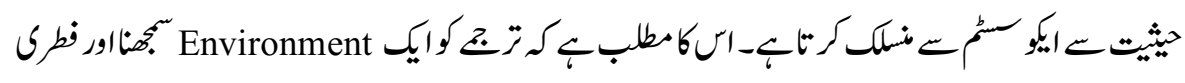

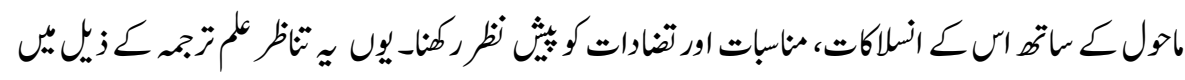

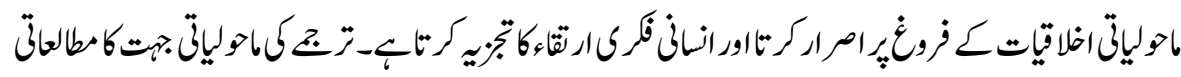

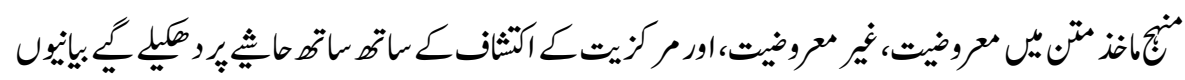

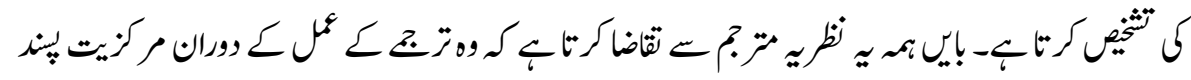

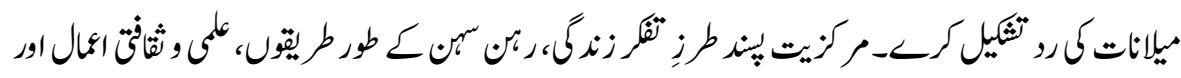

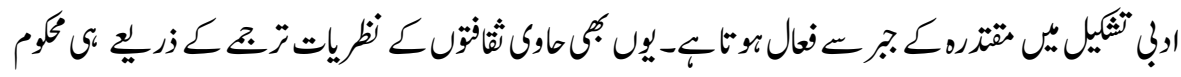

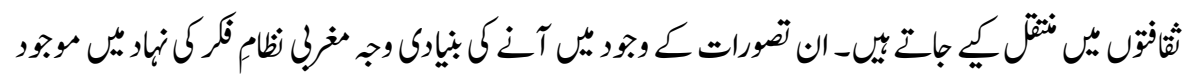




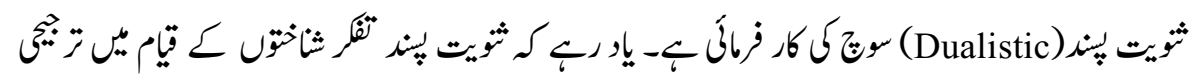

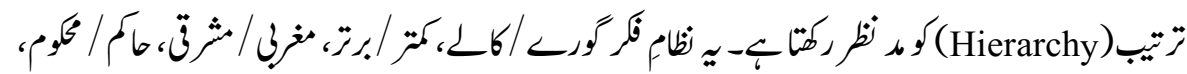

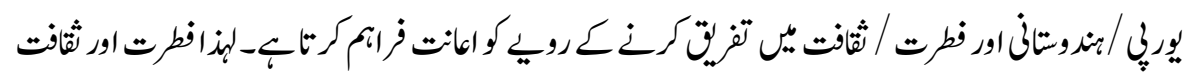

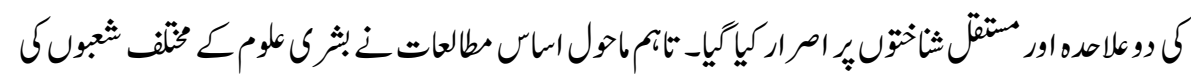

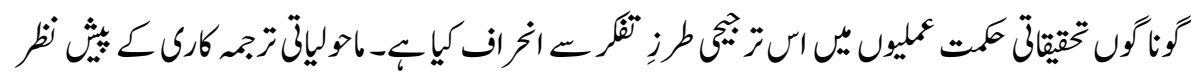

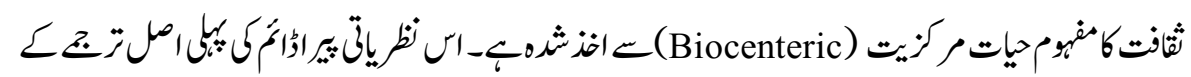

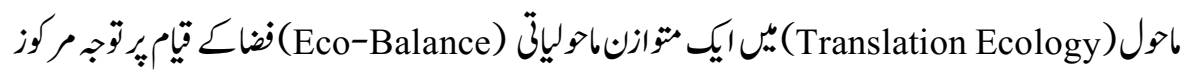

$$
\text { كر }
$$

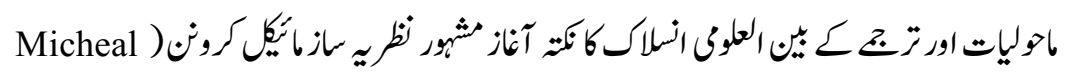

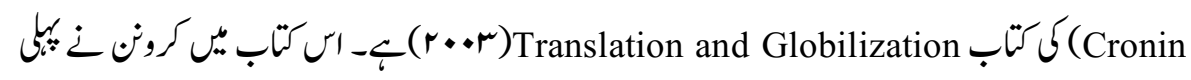

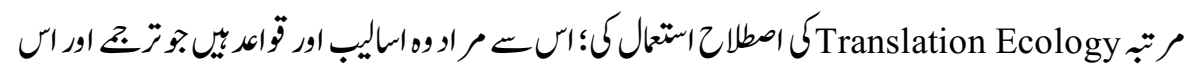

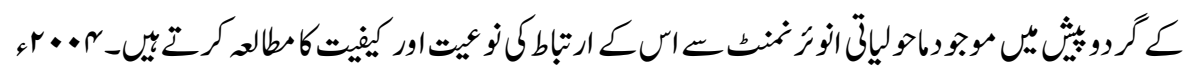

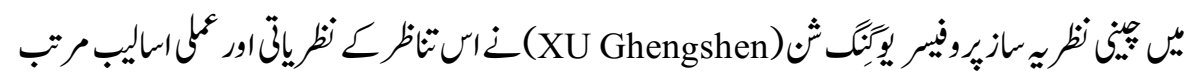

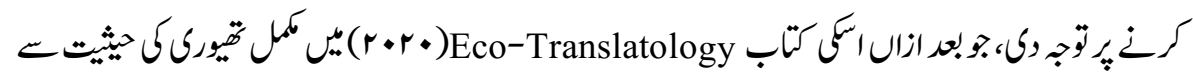

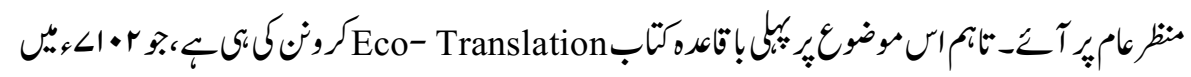

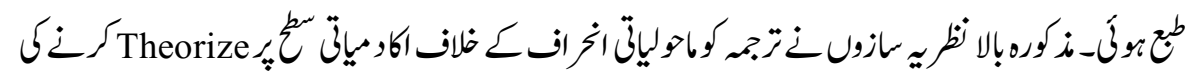
ستك

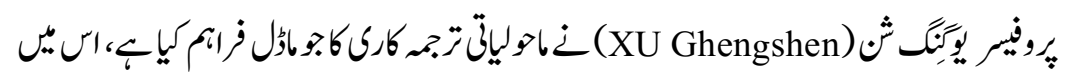

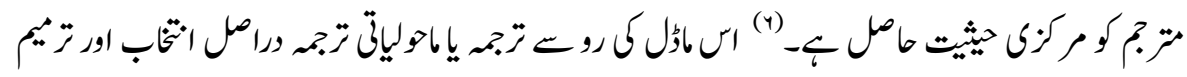
(Translation via Adaptation and Selection)

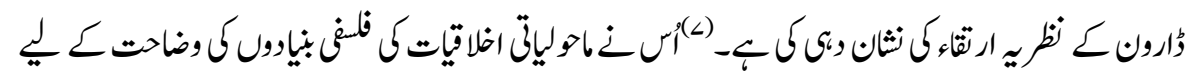

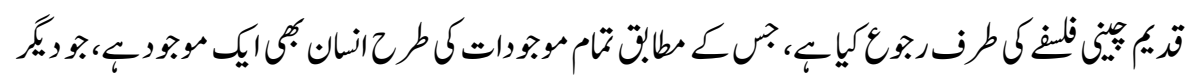

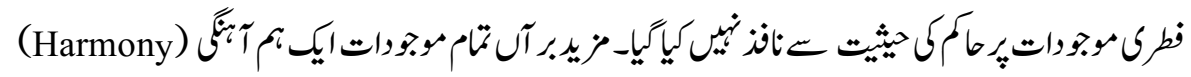




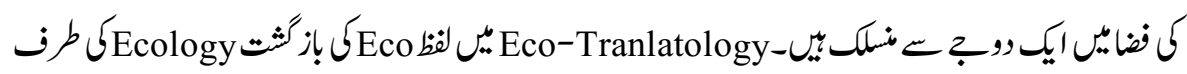

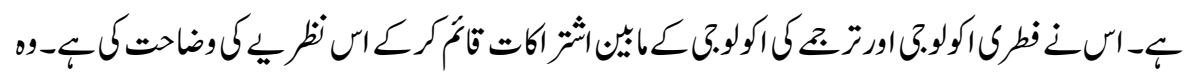

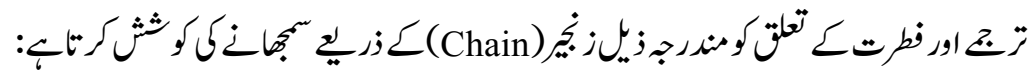

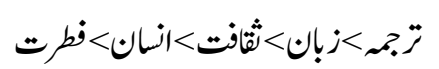

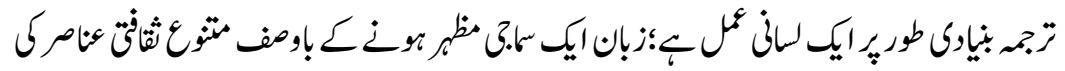

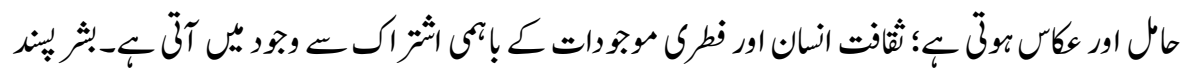

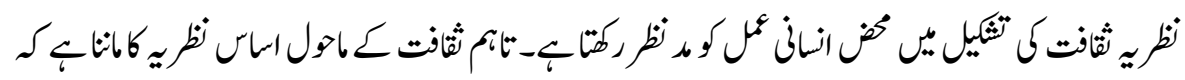

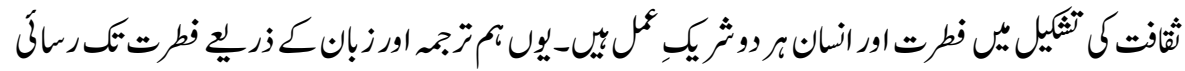

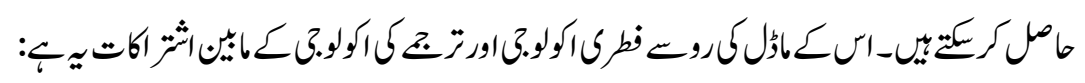

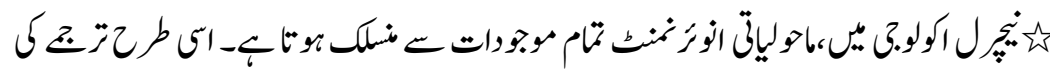

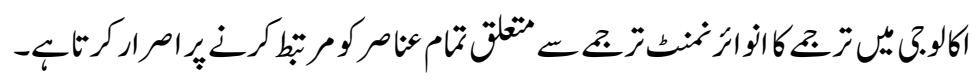

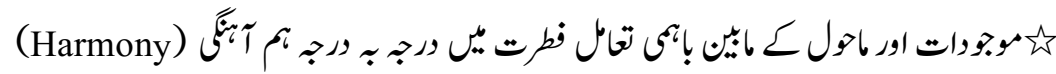

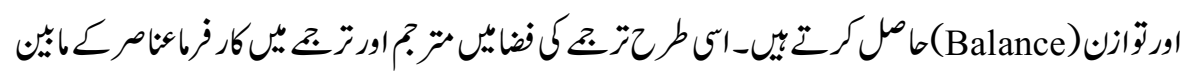

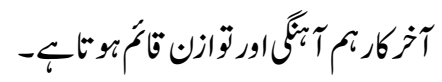

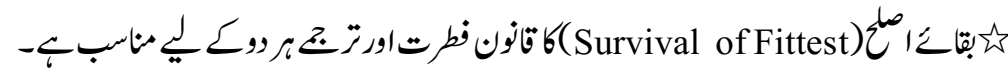

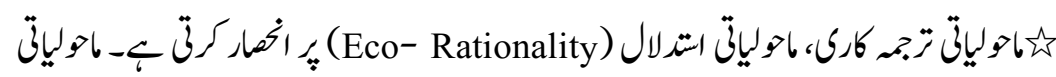

$$
\text { اتترلالتعمرا: }
$$

$$
\begin{aligned}
& \text { (Wholeness) كورصيان بي ركنا }
\end{aligned}
$$

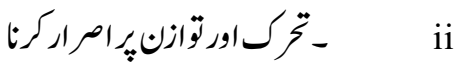

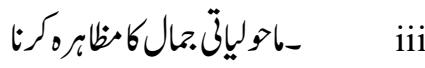

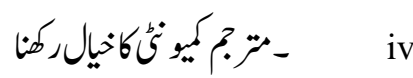

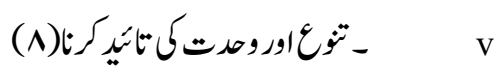




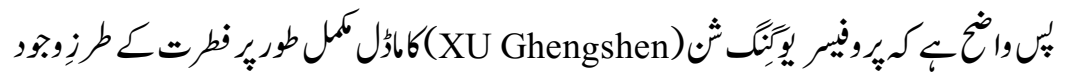

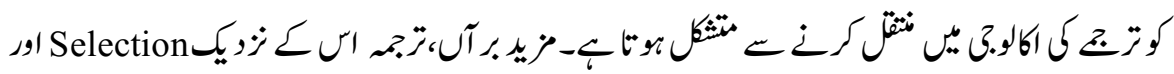
Adaptation

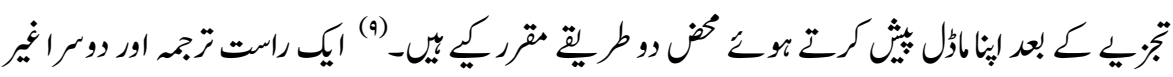

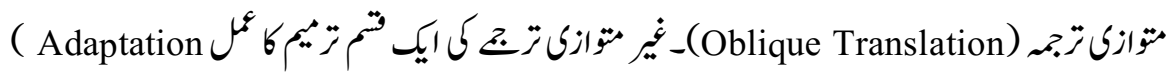

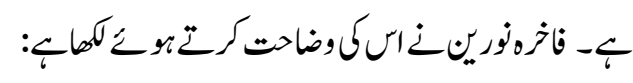

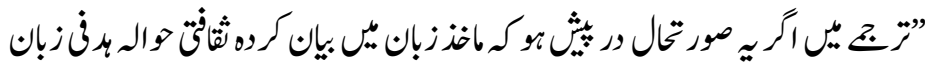

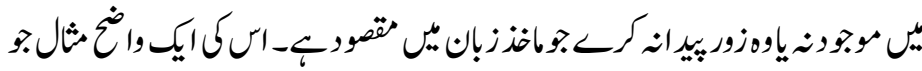

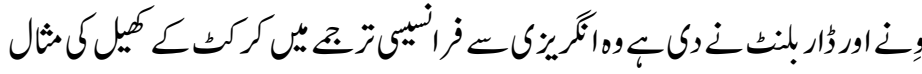

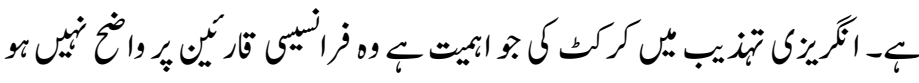

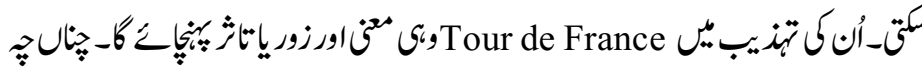

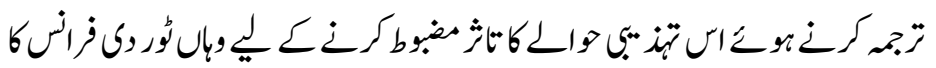

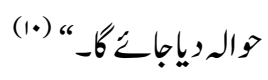

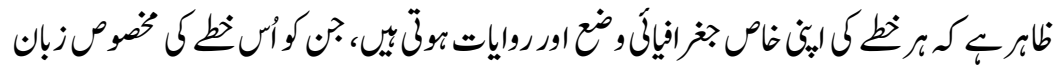

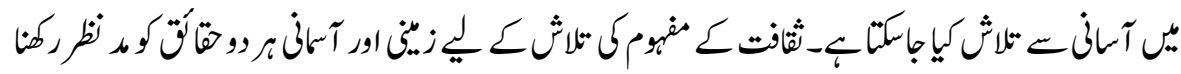

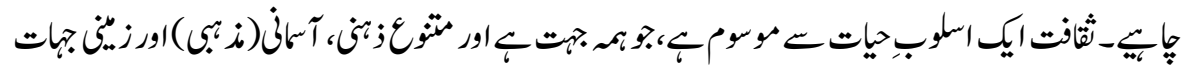

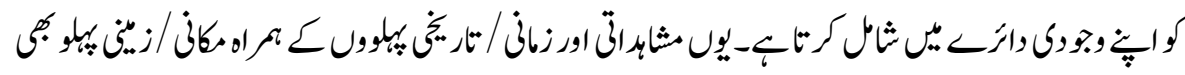

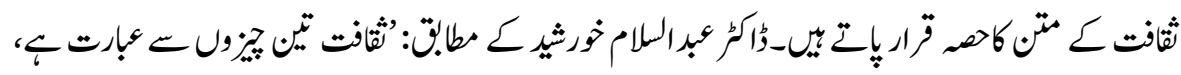

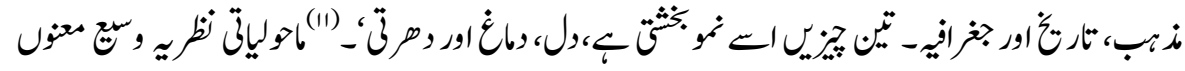

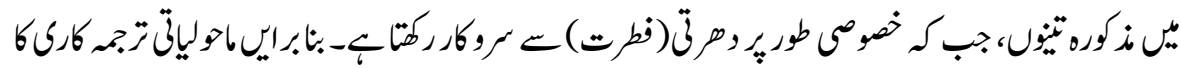

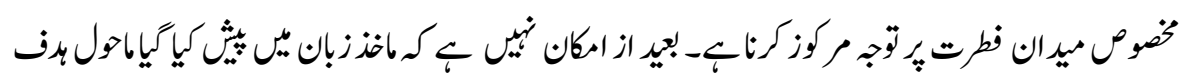

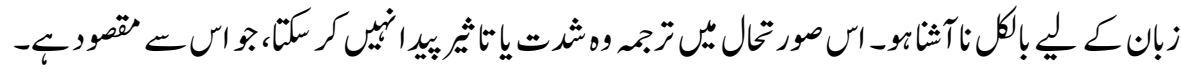

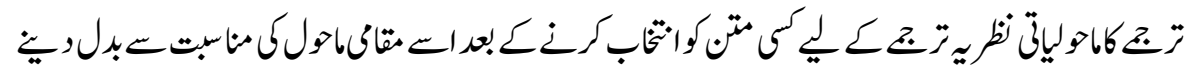




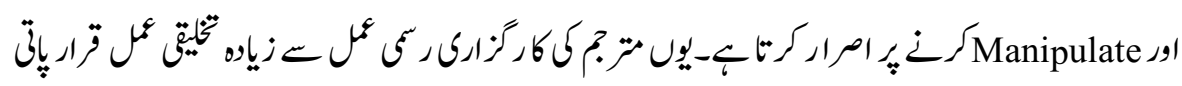

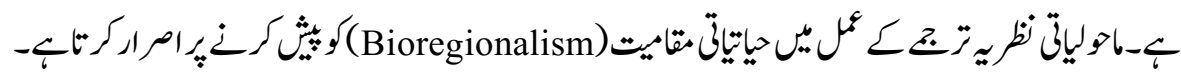

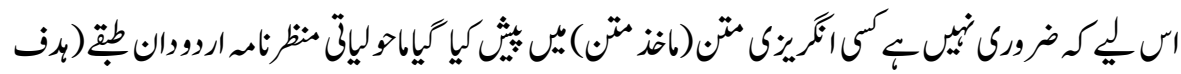

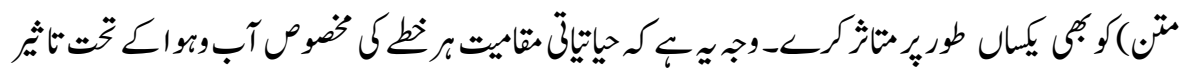

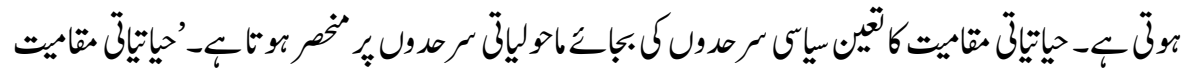

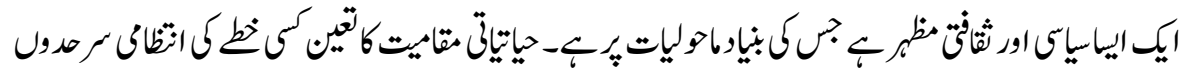

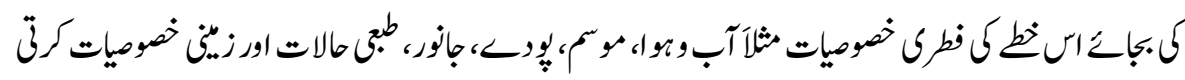

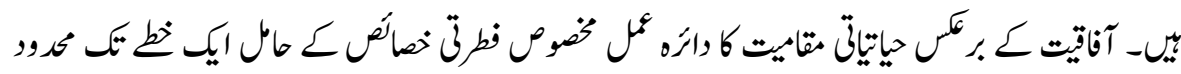

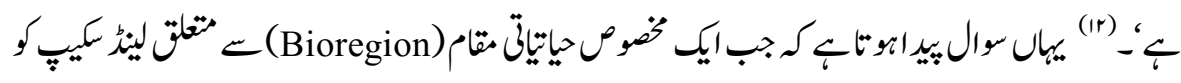

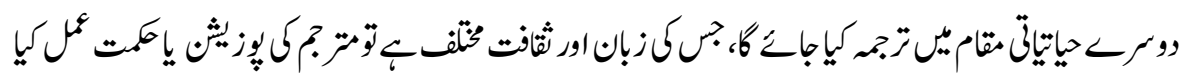

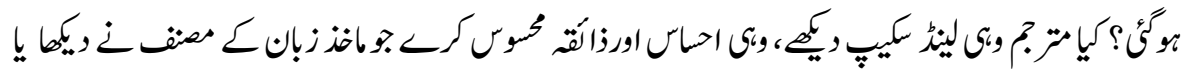

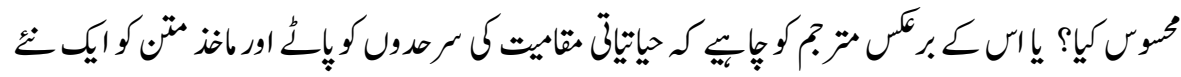

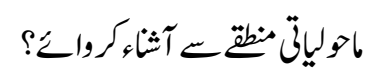

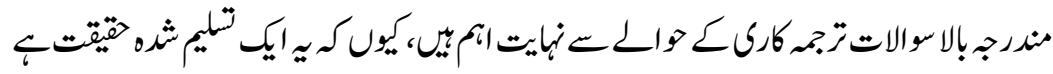

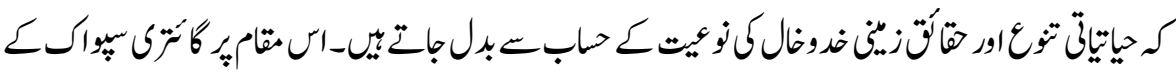

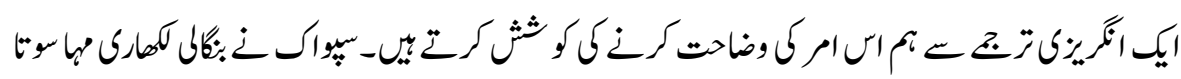

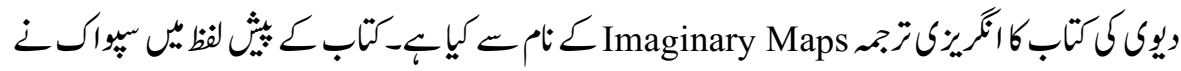

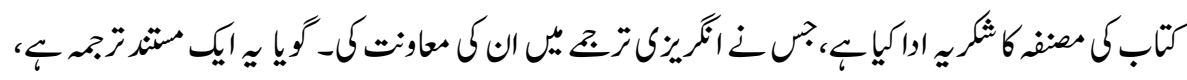

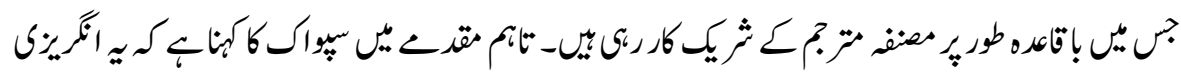

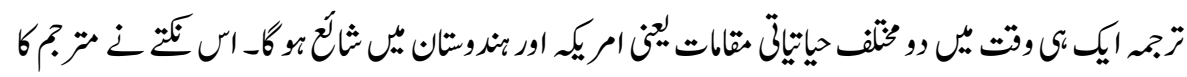

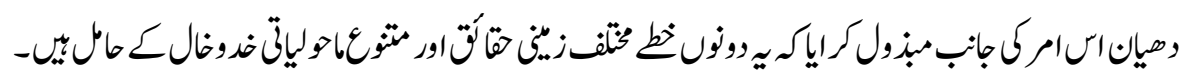

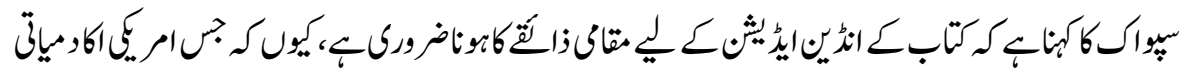

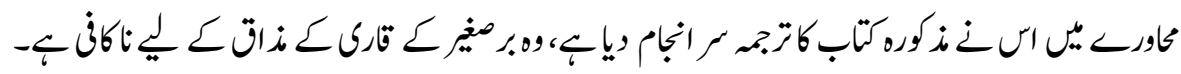




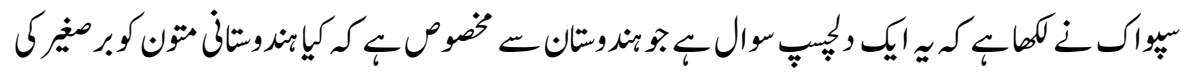

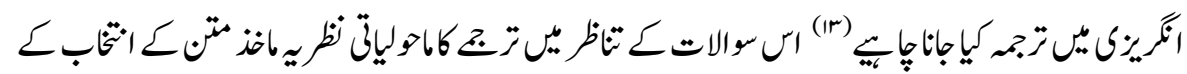

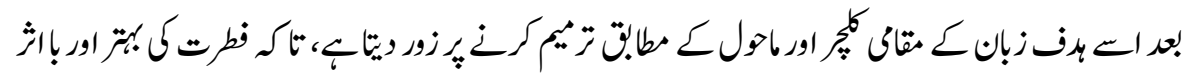

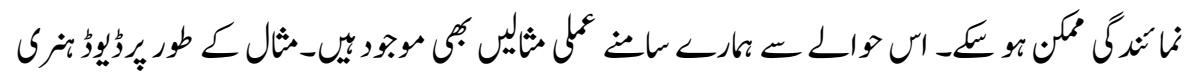

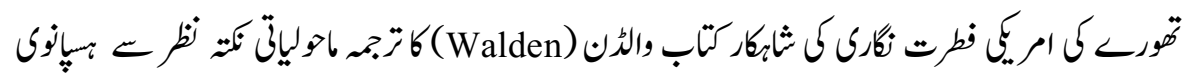

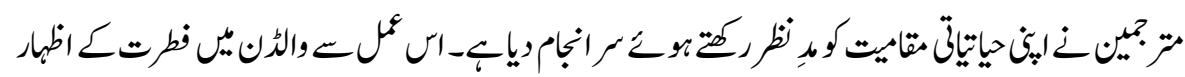

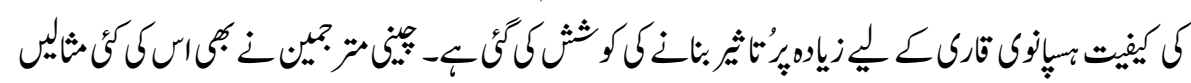

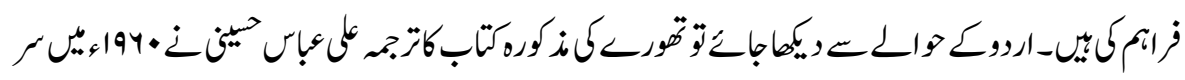

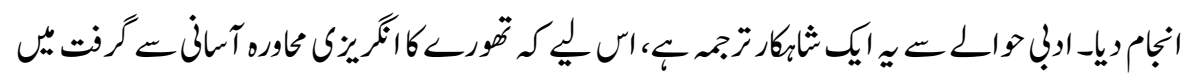

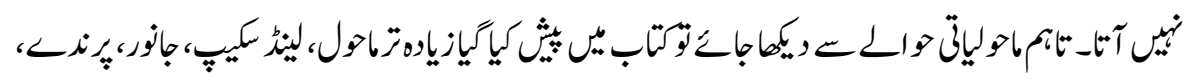

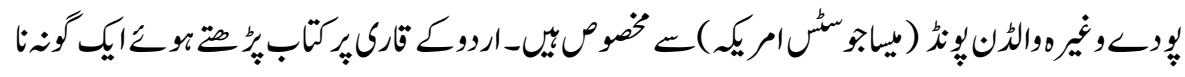

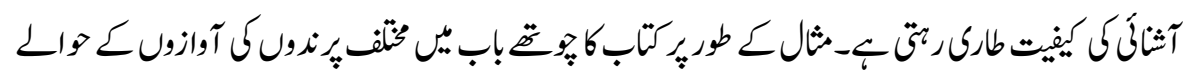

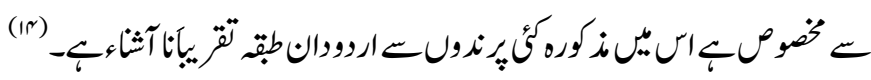

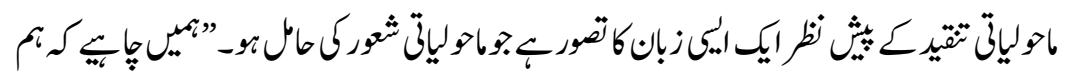

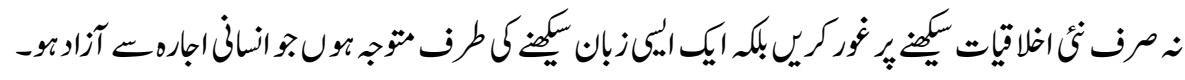

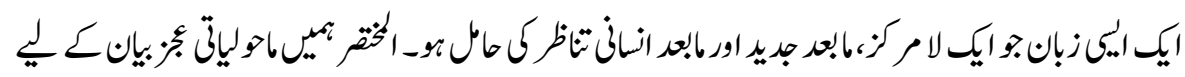

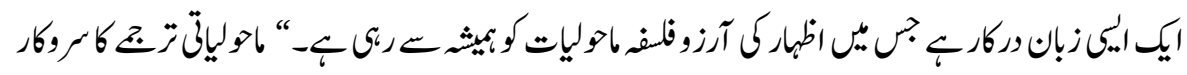

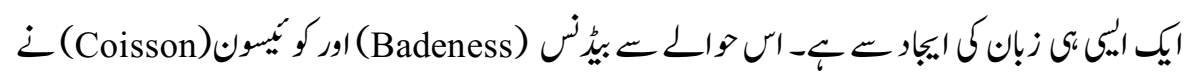

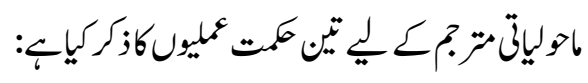

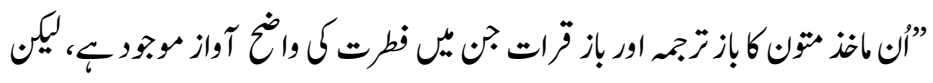

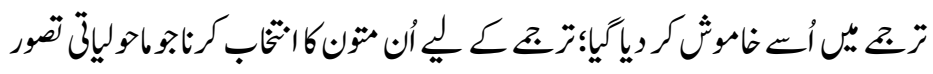

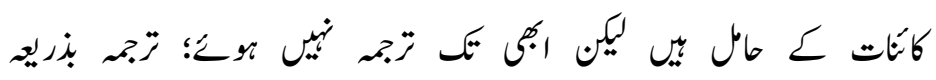


Manipulation

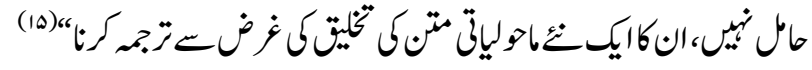

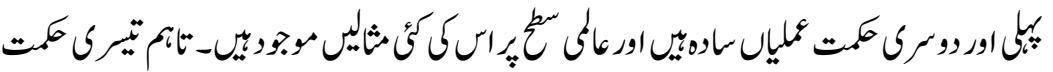

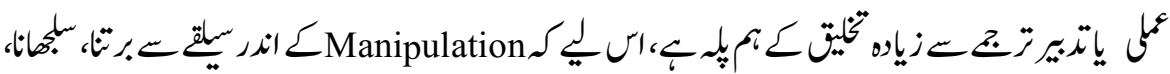

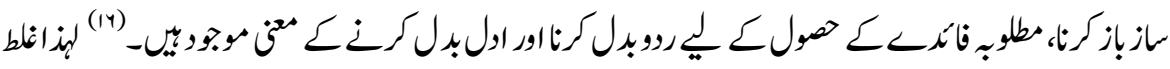

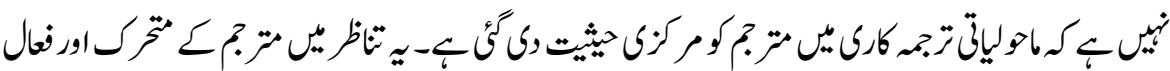

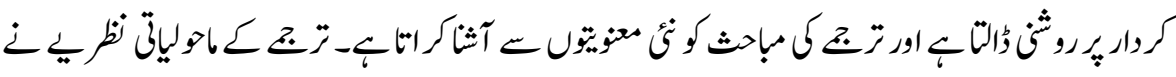

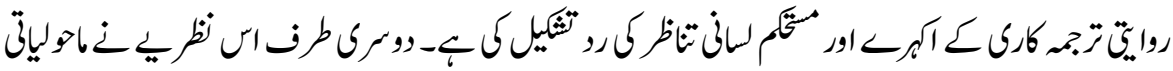

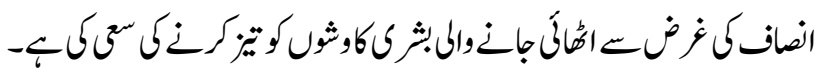

و توالرجات

Wroster, D. 'The two cultures Revised: Environmental History and the -1 environment science', Evvironment and History 2, No 1, lammi

Symposium special issue (Feb, 1996)

Catford, c. A linguistic theory of trnaslation (London: oxford

university press, 1965), 20

Doyle, M Scott. Translation and the space between: operative $-\boldsymbol{r}$ parameters of an Enterprised. In Translation: Theory and practice, Tension and Interdependence (Amsterdam: John Banjamines Pub, co,

$$
\begin{aligned}
& \text { 2008)13-26 }
\end{aligned}
$$

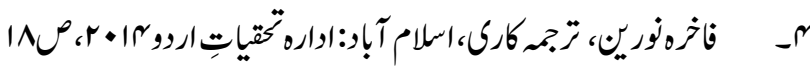

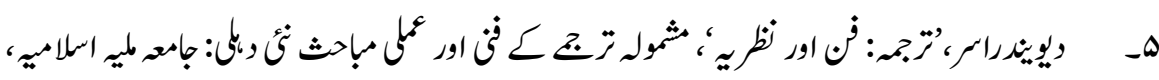

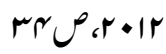

$\mathrm{Hu}$, Gengshen. Eco-Translatology(Singapore: Springer, 2020), $142 \quad-4$ 
ibid,34 - -

Hu,Gengshen. "Eco -Translatology: Research Foci and Theoretical -^

Thenets", IN chinese Translators Journal 2: 5-9

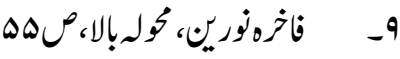

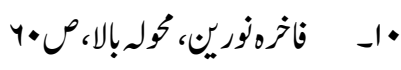

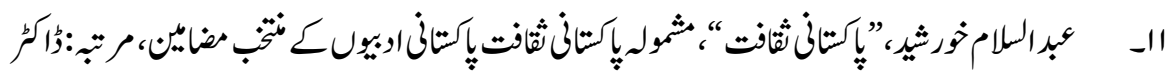

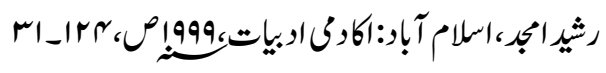

Spivak, G." Translator note ", " Translator preface". Three stories -12

Imaginary Maps (New York: Routledge, 1995, xxv-xxix

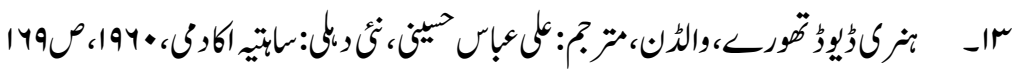

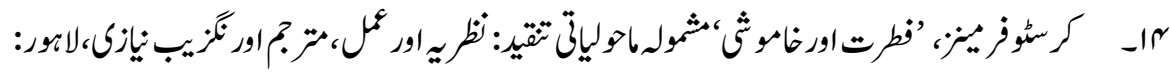

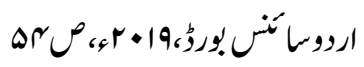

Badeness, Coisson. Ecotranslation: A Journey into the wild through the $-1 \Delta$ road less traveled. European scientific Journal 11:356-69

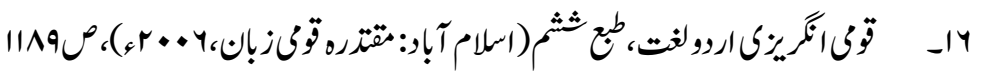

